Теорія Ймовір. та Матем. Статист. Вип. 68, 2003
Theor. Probability and Math. Statist. S 0094-9000(04)00600-3

Article electronically published on May 24, 2004

\title{
PROPERTIES OF MAXIMUM LIKELIHOOD ESTIMATES IN DIFFUSION AND FRACTIONAL-BROWNIAN MODELS
}

UDC 519.21

\author{
NADIYA RUDOMINO-DUSYATS'KA
}

\begin{abstract}
A mixed Brownian-fractional-Brownian model is considered. Two estimates for the shift parameter are constructed and compared. The local asymptotic normality and asymptotic efficiency of the estimates are established for the pure linear Brownian and fractional-Brownian models.
\end{abstract}

\section{Estimates of the Shift PARAMEter in A MiXed BROWNIAN-FRACTIONAL-BROWNIAN DIFFUSION MODEL WHERE $W_{t}$ AND $B_{t}^{H}$ ARE INDEPENDENT}

Recall that $\left(B_{t}^{H}, \mathcal{F},\left(\mathcal{F}_{t}\right)_{t \geq 0}, \mathrm{P}\right)$ is the fractional Brownian motion with Hurst index $H \in\left(\frac{1}{2} ; 1\right)$ if:

(A1) $B_{t}^{H}$ has stationary increments;

(A2) $B_{0}^{H}=0$ and $\mathrm{E} B_{t}^{H}=0$ for all $t>0$;

(A3) $\mathrm{E}\left(B_{t}^{H}\right)^{2}=t^{2 H}$ for all $t>0$;

(A4) $B_{t}^{H}$ is a Gaussian process;

(A5) $B_{t}^{H}$ has continuous trajectories.

Assume that $B_{t}^{H}$ is defined on a probability space $(\Omega, \mathcal{F}, \mathrm{P})$ and denote by $\left(\mathcal{F}_{t}\right)_{t>0}$ the filtration generated by $B_{t}^{H}$. Let the diffusion equation contain stochastic differentials with respect to the fractional Brownian motion and the Wiener process,

$$
d X_{t}=\theta X_{t} d t+\sigma_{1} X_{t} d W_{t}+\sigma_{2} X_{t} d B_{t}^{H}
$$

$X_{t=0}=X_{0} \in \mathbf{R}, 0 \leq t \leq T, T>0,\left\{\theta, \sigma_{1}, \sigma_{2}\right\} \subset \mathbf{R} \backslash\{0\}$. The construction of the stochastic differential with respect to the fractional Brownian motion is given in [1.

Assume that the processes $W_{t}$ and $B_{t}^{H}$ are independent (the case of a special dependence of $W_{t}$ and $B_{t}^{H}$ is considered in [2]). Let $\theta$ be the parameter to be estimated.

We introduce two probability measures $\widehat{Q}(t)$ and $\widetilde{Q}(t)$ as follows. The probability measure $\widehat{Q}(t)$ is determined by the following condition:

$$
\frac{d \widehat{Q}(t)}{d P(t)}=\exp \left\{\int_{0}^{t} \psi_{s} d \widehat{W}_{s}-\frac{1}{2} \int_{0}^{t} \psi_{s}^{2} d s\right\}
$$

for a nonrandom function $\psi_{s}$ such that $\mathrm{E} \int_{0}^{t} \psi_{s}^{2} d s<\infty$ and

$$
\mathrm{E}\left[\exp \left\{\int_{0}^{t} \psi_{s} d \widehat{W}_{s}-\frac{1}{2} \int_{0}^{t} \psi_{s}^{2} d s\right\}\right]=1
$$

2000 Mathematics Subject Classification. Primary 60H10, 62F12.

Key words and phrases. Fractional Brownian motion, Wiener process, diffusion model, Girsanov theorem, likelihood ratio. 
According to the Girsanov theorem,

$$
\widehat{W}_{t}=W_{t}-\int_{0}^{t} \psi_{s} d s
$$

is a standard Wiener process with respect to the probability measure $\widehat{Q}(t)$.

Let $\widetilde{Q}(t)$ be another probability measure satisfying the relation

$$
\frac{d \widetilde{Q}(t)}{d P(t)}=\exp \left\{\int_{0}^{t} s^{H-1 / 2} \delta_{s} d \widetilde{W}_{s}-\frac{1}{2} \int_{0}^{t} s^{2 H-1} \delta_{s}^{2} d s\right\},
$$

where $\delta_{s}$ is such that

$$
\int_{0}^{t} K(t, s)\left|\delta_{s}\right| d s<\infty, \quad t \in[0, T]
$$

for a kernel $K(t, s)=C_{0} s^{1 / 2-H}(t-s)^{1 / 2-H} \chi\{s \in(0 ; t)\}$. Assume that $\delta_{s}$ admits the following integral representation:

$$
\int_{0}^{t} K(t, s) \varphi_{s} d s=\int_{0}^{t} \delta_{s} d s
$$

and let the Wiener process $\widetilde{W}_{t}$ be given by the equation

$$
\int_{0}^{t} K(t, s) d B_{s}^{H}=\int_{0}^{t} s^{1 / 2-H} d \widetilde{W}_{s} .
$$

The process

$$
\widetilde{B}_{t}^{H}:=B_{t}^{H}-\int_{0}^{t} \varphi_{s} d s
$$

is a fractional Brownian motion on $[0, T]$ with respect to the measure $\widetilde{Q}(t)$ by an analogue of the Girsanov theorem for fractional Brownian motions ([3, Theorem 2.1]; see also [4]).

Excluding the shift $\theta X_{t} d t$, the total shift is

$$
\sigma_{1} \int_{0}^{t} \psi_{s} d s+\sigma_{2} \int_{0}^{t} \varphi_{s} d s=-\theta t
$$

or

$$
\sigma_{1} \psi_{t}+\sigma_{2} \varphi_{t}=-\theta
$$

Since $W_{t}$ and $B_{t}^{H}$ are independent, the final probability measure $Q(t)$ is the product of the measures $\widehat{Q}(t)$ and $\widetilde{Q}(t)$. Thus the final likelihood ratio is

$$
\begin{aligned}
& \frac{d Q(t)}{d P(t)}=\exp \left[\left\{\int_{0}^{t} \psi_{s} d \widehat{W}_{s}-\frac{1}{2} \int_{0}^{t} \psi_{s}^{2} d s\right\}\right. \\
& \left.\times\left\{\int_{0}^{t} s^{H-1 / 2} \delta_{s} d \widetilde{W}_{s}-\frac{1}{2} \int_{0}^{t} s^{2 h-1} \delta_{s}^{2} d s\right\}\right] \\
& =\exp \left\{\int_{0}^{t} \psi_{s} d \widehat{W}_{s}+\int_{0}^{t} s^{H-1 / 2} \delta_{s} d \widetilde{W}_{s}-\frac{1}{2} \int_{0}^{t}\left[\psi_{s}^{2}+s^{2 h-1} \delta_{s}^{2}\right] d s\right\} .
\end{aligned}
$$

Solving equations (4I) and (22) with respect to the functions $\psi_{t}$ and $\delta_{t}$, respectively, we obtain

$$
\begin{gathered}
\psi_{t}=-\frac{1}{\sigma_{1}}\left(\theta+\sigma_{2} \varphi_{t}\right), \\
\delta_{t}=\left(\int_{0}^{t} K(t, s) \varphi_{s} d s\right)_{t}^{\prime} .
\end{gathered}
$$


Substituting equalities (6) and (7) into likelihood ratio (5), we get that at the point $t=T$

$$
\begin{aligned}
\frac{d Q(T)}{d P(T)}=\exp \{ & -\frac{1}{\sigma_{1}} \int_{0}^{T}\left(\theta+\sigma_{2} \varphi_{s}\right) d \widehat{W}_{s}+\int_{0}^{T} s^{H-1 / 2}\left(\int_{0}^{s} K(s, u) \varphi_{u} d u\right)_{s}^{\prime} d \widetilde{W}_{s} \\
& \left.-\frac{1}{2} \int_{0}^{T}\left[\frac{1}{\sigma_{1}^{2}}\left(\theta+\sigma_{2} \varphi_{s}\right)^{2}+s^{2 H-1}\left(\left(\int_{0}^{s} K(s, u) \varphi_{u} d u\right)_{s}^{\prime}\right)^{2}\right] d s\right\} .
\end{aligned}
$$

If follows from (8) that the maximum likelihood estimate $\widehat{\theta}_{T}^{1}$ of the parameter $\theta$ satisfies the equality

$$
-\frac{1}{\sigma_{1}} \int_{0}^{T} d \widehat{W}_{s}-\frac{1}{\sigma_{1}^{2}} \int_{0}^{T}\left(\sigma_{2} \varphi_{s}+\theta\right) d s=0
$$

that can be rewritten as follows:

$$
\sigma_{1} \widehat{W}_{T}+\sigma_{2} \int_{0}^{T} \varphi_{s} d s+\theta T=0 .
$$

This gives us the following estimate of the parameter $\theta$ :

$$
\widehat{\theta}_{T}^{1}=-\frac{\sigma_{1} \widehat{W}_{T}}{T}-\frac{\sigma_{2} \int_{0}^{T} \varphi_{s} d s}{T} .
$$

Now we solve equation (4) with respect to the function $\varphi_{t}$ and substitute it into equation (9):

$$
\widehat{\theta}_{T}^{1}=-\frac{\sigma_{1} \widehat{W}_{T}}{T}-\frac{\int_{0}^{T}\left(-\theta-\sigma_{1} \psi_{s}\right) d s}{T}
$$

whence

$$
\widehat{\theta}_{T}^{1}=\theta+\frac{\sigma_{1}}{T}\left(\int_{0}^{T} \psi_{s} d s-\widehat{W}_{T}\right) .
$$

Substituting (11) into (10) yields

$$
\widehat{\theta}_{T}^{1}=\theta-\sigma_{1} \frac{W_{T}}{T} .
$$

It is evident that the estimate (11) of the parameter $\theta_{T}^{1}$ is strongly consistent.

There is another estimate of the parameter $\theta$. Solving (4) we determine the function $\varphi_{t}$. The function $\delta_{t}$ is expressed via $\varphi_{t}$ by equality (2):

$$
\begin{gathered}
\varphi_{t}=-\frac{1}{\sigma_{2}}\left(\theta+\sigma_{1} \psi_{t}\right), \\
\delta_{t}=\left(\int_{0}^{t} K(t, s) \varphi_{s} d s\right)_{t}^{\prime}=-\frac{1}{\sigma_{2}}\left(\int_{0}^{t} K(t, s)\left(\theta+\sigma_{1} \psi_{s}\right) d s\right)_{t}^{\prime} \\
=-\frac{\theta}{\sigma_{2}}\left(\int_{0}^{t} K(t, s) d s\right)_{t}^{\prime}-\frac{\sigma_{1}}{\sigma_{2}}\left(\int_{0}^{t} K(t, s) \psi_{s} d s\right)_{t}^{\prime} \\
=-\frac{\theta}{\sigma_{2}} C_{0}(2-2 H) t^{1-2 H} B\left(\frac{3}{2}-H, \frac{3}{2}-H\right)-\frac{\sigma_{1}}{\sigma_{2}}\left(\int_{0}^{t} K(t, s) \psi_{s} d s\right)_{t}^{\prime} .
\end{gathered}
$$

Set $B_{1}=B\left(\frac{3}{2}-H, \frac{3}{2}-H\right)$. Using equalities (6) and (17) for the likelihood ratio (5), taking the logarithms, differentiating with respect to $\theta$, and equating the derivative to 
zero, we obtain that at the point $t=T$

$$
\begin{aligned}
& \frac{C_{0} B_{1}(2-2 H)}{\sigma_{2}} \int_{0}^{T} s^{1 / 2-H} d \widetilde{W}_{s} \\
& +\int_{0}^{T} s^{2 H-1}\left[\theta\left(\frac{C_{0} B_{1}(2-2 H)}{\sigma_{2}}\right)^{2} s^{2-4 H}\right. \\
& \left.\quad+\frac{C_{0} B_{1}(2-2 H)}{\sigma_{2}} s^{1-2 H}\left(\int_{0}^{s} K(T, u) \psi_{u} d u\right)_{s}^{\prime}\right] d s=0,
\end{aligned}
$$

or

$$
\int_{0}^{T} s^{1 / 2-H} d \widetilde{W}_{s}+\theta \frac{C_{0} B_{1}}{\sigma_{2}} T^{2-2 H}+\frac{\sigma_{1}}{\sigma_{2}} \int_{0}^{T} K(T, s) \psi_{s} d s=0 .
$$

This implies another estimate for the parameter $\theta$ :

$$
\widehat{\theta}_{T}^{2}=\frac{-\sigma_{2} \int_{0}^{T} s^{1 / 2-H} d \widetilde{W}_{s}-\sigma_{1} \int_{0}^{T} K(T, s) \psi_{s} d s}{C_{0} B_{1} T^{2-2 H}} .
$$

Now we substitute expression (6) for the function $\psi_{t}$ into relation (14) and obtain

$$
\begin{aligned}
\widehat{\theta}_{T}^{2} & =-\frac{\sigma_{2}}{C_{0} B_{1} T^{2-2 H}} \int_{0}^{T} s^{1 / 2-H} d \widetilde{W}_{s}+\frac{1}{C_{0} B_{1} T^{2-2 H}} \int_{0}^{T} K(T, s)\left(\theta+\sigma_{2} \varphi_{s}\right) d s \\
& =\theta+\frac{\sigma_{2}}{C_{0} B_{1} T^{2-2 H}}\left[\int_{0}^{T} K(T, s) \varphi_{s} d s-\int_{0}^{T} s^{1 / 2-H} d \widetilde{W}_{s}\right] .
\end{aligned}
$$

Recall that $\int_{0}^{T} s^{1 / 2-H} d \widetilde{W}_{s}=\int_{0}^{T} K(T, s) d B_{s}^{H}$. Thus

$$
\begin{array}{rl}
\int_{0}^{T} & K(T, s) \varphi_{s} d s-\int_{0}^{T} K(T, s) d B_{s}^{H} \\
& =\int_{0}^{T} K(T, s) \varphi_{s} d s-\int_{0}^{T} K(T, s) d\left(\widetilde{B}_{s}^{H}+\int_{0}^{T} \varphi_{s} d s\right)=-\int_{0}^{T} K(T, s) d \widetilde{B}_{s}^{H} .
\end{array}
$$

The second estimate of the parameter $\theta$ is given by

or

$$
\widehat{\theta}_{T}^{2}=\theta-\frac{\sigma_{2}}{C_{0} B_{1} T^{2-2 H}} \int_{0}^{T} K(T, s) d \widetilde{B}_{s}^{H},
$$

$$
\widehat{\theta}_{T}^{2}=\theta-\frac{\sigma_{2}}{C_{0} B_{1}(2-2 H)} \frac{\int_{0}^{T} s^{1 / 2-H} d \widetilde{W}_{s}}{\frac{T^{2-2 H}}{2-2 H}} .
$$

The strong consistency of the estimate $\widehat{\theta}_{T}^{2}$ is also clear.

Now we compare the estimates $\widehat{\theta}_{T}^{1}$ and $\widehat{\theta}_{T}^{2}$.

First we compute the variances of the remainder terms in formulas (11) and (15) and compare

$$
\frac{\sigma_{1}}{T} \quad \text { and } \quad \frac{\sigma_{2}^{2}}{C_{0}^{2} B_{1}^{2}(2-2 H)} \frac{1}{T^{2-2 H}} .
$$

Since $H \in\left(\frac{1}{2} ; 1\right)$, it is obvious that there exists a number $N$ such that

$$
\frac{\sigma_{1}}{T}<\frac{\sigma_{2}^{2}}{C_{0}^{2} B_{1}^{2}(2-2 H)} \frac{1}{T^{2-2 H}}
$$


for all $T>N$. This means that the variance of the deviation of the estimate $\widehat{\theta}_{T}^{1}$ from the true value is smaller than that of the corresponding deviation of the estimate $\widehat{\theta}_{T}^{2}$ from the true value. It this sense, the estimate $\widehat{\theta}_{T}^{1}$ is better than $\widehat{\theta}_{T}^{2}$.

2. LOCAL ASYMPTOTIC NORMALITY AND ASYMPTOTIC EFFICIENCY OF THE ESTIMATE OF THE SHIFT PARAMETER IN A LINEAR BROWNIAN DIFFUSION MODEL

Consider a pure linear Brownian model

$$
d X_{t}=\frac{1}{T^{\alpha}} \theta X_{t} d t+c X_{t} d W_{t}, \quad X_{t=0}=X_{0}, \theta \in \mathbf{R}, t \in[0, T], \alpha \in\left(\frac{1}{2}, 1\right] .
$$

Put $\Theta=(0, \infty), \theta \in \Theta$. According to Definition 2.1 in [5], a family of measures $P_{\theta}(t)$ is locally asymptotically normal (LAN) at the point $\theta \in \Theta$ as $t \rightarrow \infty$ if

$$
Z_{t, \theta}(u)=\frac{d P_{\theta+A(t, \theta) u}(t)}{d P_{\theta}(t)}=\exp \left\{u \xi_{t, \theta}-\frac{1}{2} u^{2}+\zeta_{t}(u, \theta)\right\}
$$

for some function $A(t, \theta)$ and any number $u \in \mathbf{R}$, where $\xi_{t, \theta} \Rightarrow N(0,1)$ as $t \rightarrow \infty$ with respect to the measure $P_{\theta}(t)$, and $\zeta_{t}(u, \theta) \stackrel{\mathrm{P}}{\rightarrow} 0, t \rightarrow \infty$, for all numbers $u \in \mathbf{R}$. We say in this case that the LAN property holds for the family of measures $P_{\theta}(t)$ as $t \rightarrow \infty$ at the point $\theta$.

Theorem 1. The LAN property holds for the family of measures $P_{\theta}(t)$ as $t \rightarrow \infty$ at any point $\theta \in \Theta$.

Proof. We change the probability measure $P_{\theta}(t)$ for the measure $P_{0}(t)$. Then the shift $\theta X_{t} d t$ disappears and we obtain

$$
X_{t}=X_{0}+\frac{\theta}{T^{\alpha}} \int_{0}^{t} X_{s} d s+c \int_{0}^{t} X_{s} d W_{s}=X_{0}+c \int_{0}^{t} X_{s} d\left(W_{s}+\frac{\theta}{c T^{\alpha}} s\right) .
$$

This change of measure transforms the Wiener process $W_{t}+t \theta /\left(c T^{\alpha}\right)$ into the new process $\widehat{W}_{t}$, and $\int_{0}^{t} \varphi_{s} d s=t \theta /\left(c T^{\alpha}\right)$, that is, $\varphi_{t}=\theta /\left(c T^{\alpha}\right)$.

Consider the likelihood ratio corresponding to this change of measure

$$
\begin{aligned}
\frac{d P_{\theta}(t)}{d P_{0}(t)} & =\exp \left\{\int_{0}^{t} \varphi_{s} d \widehat{W}_{s}-\frac{1}{2} \int_{0}^{t} \varphi_{s}^{2} d s\right\}=\exp \left\{\int_{0}^{t} \frac{\theta}{c T^{\alpha}} d \widehat{W}_{s}-\frac{1}{2} \int_{0}^{t} \frac{\theta^{2}}{\left(c T^{\alpha}\right)^{2}} d s\right\} \\
& =\exp \left\{\frac{\theta}{c T^{\alpha}} \widehat{W}_{t}-\frac{1}{2} \frac{\theta^{2}}{\left(c T^{\alpha}\right)^{2}} t\right\} .
\end{aligned}
$$

Now we consider the linear model with parameter $\theta$ shifted by $A(t) u$. The likelihood ratio for such a change of measure is of the form

$$
\frac{P_{\theta+A(t) u}(t)}{d P_{0}(t)}=\exp \left\{\frac{1}{c T^{\alpha}}(\theta+A(t) u) \widehat{W}_{t}-\frac{1}{2\left(c T^{\alpha}\right)^{2}}(\theta+A(t) u)^{2} t\right\}
$$

and

$$
\begin{aligned}
& \frac{d P_{\theta+A(t, \theta) u}(t)}{d P_{\theta}(t)}=\frac{d P_{\theta+A(t, \theta) u}(t)}{d P_{0}(t)} \cdot\left(\frac{d P_{\theta}(t)}{d P_{0}(t)}\right)^{-1} \\
& \quad=\exp \left\{\frac{1}{c T^{\alpha}}(\theta+A(t) u) \widehat{W}_{t}-\frac{1}{2\left(c T^{\alpha}\right)^{2}}(\theta+A(t) u)^{2} t-\frac{\theta}{c T^{\alpha}} \widehat{W}_{t}-\frac{1}{2} \frac{\theta^{2}}{\left(c T^{\alpha}\right)^{2}} t\right\} \\
& \quad=\exp \left\{\frac{u A(t)}{c T^{\alpha}} \widehat{W}_{t}-\frac{1}{2} u^{2} \frac{A^{2}(t)}{\left(c T^{\alpha}\right)^{2}} t-\frac{A(t) u \theta}{\left(c T^{\alpha}\right)^{2}} t\right\} .
\end{aligned}
$$


Set $A(t)=c T^{\alpha} / \sqrt{t}$. Then

$$
\frac{d P_{\theta+A(t, \theta) u}(t)}{d P_{\theta}(t)}=\exp \left\{u \frac{\widehat{W}_{t}}{\sqrt{t}}-\frac{1}{2} u^{2}-\frac{u \theta \sqrt{t}}{c T^{\alpha}}\right\} .
$$

Since $\widehat{W}_{t} / \sqrt{t} \Rightarrow N(0,1)$ and $u \theta \sqrt{t} /\left(c T^{\alpha}\right) \rightarrow 0$ as $t \rightarrow \infty$ for $T \geq t$ and $\alpha>\frac{1}{2}$, the above definition implies the LAN property for the family $P_{\theta}(t)$ as $t \rightarrow \infty$ and at any point $\theta \in \Theta$.

Now we are able to prove the asymptotic efficiency of the estimate $\left\{\theta_{t}, t>0\right\}$. According to the definition introduced in the monograph [5], an estimate $\left\{\theta_{t}, t>0\right\}$ of a parameter $\theta$ is asymptotically efficient under the LAN property for the cost function $\omega\left(A^{-1}(t, \theta) x\right)$ at the point $\theta$ if

$$
\lim _{\delta \rightarrow 0} \lim _{t \rightarrow \infty} \sup _{\left|\theta^{\prime}-\theta\right|<\delta} \mathrm{E}_{P_{\theta^{\prime}}(t)} \omega\left(A^{-1}(t, \theta)\left(\theta_{t}-\theta^{\prime}\right)\right)=\mathrm{E} \omega(N(0,1)) .
$$

Let $\omega \in W$, where $W$ is the class of functions defined on $\Theta$ and satisfying the conditions:

1) $w(u) \geq 0, w(0)=0, w$ is a Borel function continuous at zero and not identically zero;

2) $w(u)=w(-u)$

3) the set $\{u: w(u)<c\}$ is convex for any $c>0$.

The estimate $\left\{\theta_{t}, t>0\right\}$ is asymptotically efficient for the cost function

$$
w\left(A^{-1}(t, \theta) x\right) \in W_{p},
$$

where $W_{p} \subset W$ is the class of functions of $W$ that have a polynomial dominant.

Consider the maximum likelihood estimate of the parameter $\theta$ in a linear Brownian model

$$
\widehat{\theta}_{t}=\frac{c T^{\alpha}}{t} \widehat{W}_{t}=\frac{c T^{\alpha}}{t}\left(W_{t}+\frac{1}{c T^{\alpha}} \theta t\right)=\theta+\frac{c T^{\alpha}}{t} W_{t} .
$$

To prove the asymptotic efficiency of the estimate $\widehat{\theta}_{t}$ we use Theorem 1.3 in [5]. According to this theorem, the estimate $\widehat{\theta}_{t}$ is asymptotically efficient in the sense mentioned above if the following conditions hold:

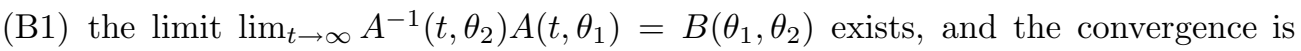
uniform in $\theta_{i} \in \Theta$

(B2) $\zeta_{t}(\theta):=A^{-1}(t, \theta)\left(\widehat{\theta}_{t}-\theta\right) \Rightarrow N(0,1)$ uniformly in $\theta_{i} \in \Theta$ as $t \rightarrow \infty$ with respect to the measure $P_{\theta}(t)$;

(B3) random variables $\left|A^{-1}(t, \theta)\left(\widehat{\theta}_{t}-\theta\right)\right|^{N}, N>0$, are $P_{\theta}(t)$-integrable for any $\theta \in \Theta$ uniformly in $t>t_{0}(N)$.

Conditions (B1) and (B3) hold in the case under consideration, since

$$
A(t)=\frac{c T^{\alpha}}{\sqrt{t}}
$$

does not depend on $\theta$.

Now we check condition (B2):

$$
\zeta_{t}(\theta)=A^{-1}(t, \theta)\left(\widehat{\theta}_{t}-\theta\right)=\frac{\sqrt{t}}{c T^{\alpha}} \frac{c T^{\alpha}}{t} W_{t}=W_{t} \frac{1}{\sqrt{t}} \Rightarrow N(0,1) .
$$

Thus the estimate $\widehat{\theta}_{t}$ is asymptotically efficient as $t \rightarrow \infty$. 
3. LoCAL ASYMPTOTIC NORMALITY AND ASYMPTOTIC EFFICIENCY OF THE ESTIMATE OF THE SHIFT PARAMETER IN A LINEAR FRACTIONAL-BROWNIAN DIFFUSION MODEL

Consider a pure linear fractional-Brownian model

$$
\begin{gathered}
d X_{t}=\frac{1}{T^{\alpha}} \theta X_{t} d t+X_{t} d B_{t}^{H}, \\
X_{t=0}=X_{0}, \quad \theta \in \mathbf{R}, \quad t \in[0, T], \quad \alpha \in(1-H, 1] .
\end{gathered}
$$

It will be clear from the further argument that it is sufficient to consider the case of

$$
\alpha \in\left(1-H, \frac{1}{2}\right)
$$

in this model. In this case $\varphi_{t}=\theta / T^{\alpha}$. Then

$$
\int_{0}^{t} \delta_{s} d s=\int_{0}^{t} K(t, s) \frac{\theta}{T^{\alpha}} d s=\frac{\theta}{T^{\alpha}} C_{0} \int_{0}^{t} s^{1 / 2-H}(t-s)^{1 / 2-H} d s=\frac{\theta}{T^{\alpha}} C_{0} B_{1} t^{2-2 H},
$$

$\delta_{t}=\left(\theta / T^{\alpha}\right) C_{0} B_{1} t^{1-2 H}(2-2 H)$. Therefore

$$
\widehat{\theta}_{t}=-\frac{T^{\alpha} \int_{0}^{t} s^{1 / 2-H} d \widehat{W}_{s}}{C_{0} B_{1} t^{2-2 H}}
$$

where

$$
\widehat{W}_{s}=W_{s}-\frac{\theta}{T^{\alpha}} C_{0} B_{1} \frac{2-2 H}{\frac{3}{2}-H} s^{3 / 2-H} .
$$

In other words,

$$
\widehat{\theta}_{t}=\theta-\frac{T^{\alpha} \int_{0}^{t} s^{1 / 2-H} d W_{s}}{C_{0} B_{1} t^{2-2 H}}
$$

Put $\Theta=(0, \infty), \theta \in \Theta$.

Theorem 2. The LAN property holds for the family $P_{\theta}(t)$ as $t \rightarrow \infty$ at any point $\theta \in \Theta$.

Proof. We replace the probability measure $P_{\theta}(t)$ with the measure $P_{0}(t)$. As a result, the shift $\theta X_{t} d t$ disappears. The corresponding likelihood ratio is given by

$$
\begin{aligned}
\frac{d P_{\theta}(t)}{d P_{0}(t)} & =\exp \left\{\int_{0}^{t} s^{H-1 / 2} \delta_{s} d \widehat{W}_{s}-\frac{1}{2} \int_{0}^{t} s^{2 H-1} \delta_{s}^{2} d s\right\} \\
& =\exp \left\{\frac{\theta C_{0} B_{1}(2-2 H)}{T^{\alpha}} \int_{0}^{t} s^{1 / 2-H} d \widehat{W}_{t}-\frac{1}{2 T^{2 \alpha}}\left(\theta C_{0} B_{1}(2-2 H)\right)^{2} \frac{t^{2-2 H}}{2-2 H}\right\} .
\end{aligned}
$$

Now we consider the linear model with parameter $\theta$ shifted by $A(t) u$. Put

$$
K:=C_{0} B_{1}(2-2 H)
$$

then

$$
\frac{P_{\theta+A(t) u}(t)}{d P_{0}(t)}=\exp \left\{\frac{(\theta+A(t) u) K}{T^{\alpha}} \int_{0}^{t} s^{1 / 2-H} d \widehat{W}_{t}-\frac{1}{2 T^{2 \alpha}}((\theta+A(t) u) K)^{2} \frac{t^{2-2 H}}{2-2 H}\right\} .
$$


The likelihood ratio for this model is of the form

$$
\begin{aligned}
& \frac{d P_{\theta+A(t, \theta) u}(t)}{d P_{\theta}(t)}=\frac{d P_{\theta+A(t, \theta) u}(t)}{d P_{0}(t)} \cdot\left(\frac{d P_{\theta}(t)}{d P_{0}(t)}\right)^{-1} \\
& =\exp \left\{\frac{K}{T^{\alpha}}(\theta+A(t) u) \int_{0}^{t} s^{1 / 2-H} d \widehat{W}_{t}-\frac{1}{2 T^{2 \alpha}}((\theta+A(t) u) K)^{2} \frac{t^{2-2 H}}{2-2 H}\right. \\
& \left.\quad-\theta \frac{K}{T^{\alpha}} \int_{0}^{t} s^{1 / 2-H} d \widehat{W}_{t}-\frac{1}{2 T^{2}}(\theta K)^{2} \frac{t^{2-2 H}}{2-2 H}\right\} \\
& =\exp \left\{\frac{K}{T^{\alpha}} A(t) u \int_{0}^{t} s^{1 / 2-H} d \widehat{W}_{t}-\frac{1}{2} u^{2} A^{2}(t) \frac{K^{2}}{T^{2 \alpha}} \frac{t^{2-2 H}}{2-2 H}-A(t) u \theta \frac{K^{2}}{T^{2 \alpha}} \frac{t^{2-2 H}}{2-2 H}\right\} .
\end{aligned}
$$

Set $A(t):=T^{\alpha} \sqrt{2-2 H} /\left(K t^{1-H}\right)$. Then the likelihood ratio takes the form

$$
\frac{d P_{\theta+A(t, \theta) u}(t)}{d P_{\theta}(t)}=\exp \left\{u \frac{\int_{0}^{t} s^{1 / 2-H} d \widehat{W}_{s}}{\frac{t^{1-H}}{\sqrt{2-2 H}}}-\frac{1}{2} u^{2}-\frac{u \theta K t^{1-H}}{T^{\alpha} \sqrt{2-2 H}}\right\} .
$$

Since

$$
\frac{\int_{0}^{t} s^{1 / 2-H} d \widehat{W}_{s}}{\frac{t^{1-H}}{\sqrt{2-2 H}}} \Rightarrow N(0,1)
$$

and

$$
\frac{u \theta K t^{1-H}}{T^{\alpha} \sqrt{2-2 H}} \rightarrow 0 \quad \text { as } t \rightarrow \infty,
$$

the LAN property holds for the family $P_{\theta}(t)$ as $t \rightarrow \infty$ at any point $\theta \in \Theta$.

Now we check the asymptotic efficiency of the estimate $\widehat{\theta}_{t}$. Consider conditions (B1)(B3). Two of them, (B1) and (B3), are evident. To check (B2) we use the following relations:

$\zeta_{t}(\theta)=A^{-1}(t, \theta)\left(\widehat{\theta}_{t}-\theta\right)=\frac{K t^{1-H}}{T^{\alpha} \sqrt{2-2 H}} \frac{T^{\alpha} \int_{0}^{t} s^{1 / 2-H} d W_{s}}{C_{0} B_{1} t^{2-2 H}}=\frac{\int_{0}^{t} s^{1 / 2-H} d W_{s}}{\frac{t^{1-H}}{\sqrt{2-2 H}}} \Rightarrow N(0,1)$.

Therefore, the estimate $\widehat{\theta}_{t}$ of the parameter $\theta$ is asymptotically efficient as $t \rightarrow \infty$.

\section{BIBLIOGRAPHY}

1. M. Zähle, Integration with respect to fractional functions and stochastic calculus. I, Probab. Theory Related Fields 111 (1998), 333-374. MR 99j:60073

2. Yu. Mishura and N. Rudomino-Dusyatska, Consistency of drift parameter estimates in fractional Brownian diffusion models, Theory Stoch. Processes 7(23) (2001), no. 3-4, 103-112.

3. A. Kukush, Yu. Mishura, and E. Valkeila, Statistical Inference with Fractional Brownian Motion, Preprint, Turku University, 2001.

4. L. Decreusefond and A. S. Üstünel, Stochastic analysis on fractional Brownian motion, Potential Analysis 10 (1998), 177-214. MR 2000b:60133

5. I. A. Ibragimov and R. Z. Khas'minskiı̌, Statistical Estimation: Asymptotic Theory, "Nauka", Moscow, 1979; English transl., Springer-Verlag, Berlin-Heidelberg-New York, 1981. MR 81h:62004

Department of Probability Theory and Mathematical Statistics, Faculty of Mechanics and Mathematics, Kyiv National Taras Shevchenko University, Academician Glushkov Avenue 6 , KYIV-127 03127, UKRAINE

E-mail address: nadiya_rudomino@hotmail.com

Received 20/JUN/2002

Translated by YU. MISHURA 